\title{
Direct Formation of Silver Cluster Ions from Silver Salts by Laser Desorption/Ionization
}

\author{
Sung-Seen Choi, ${ }^{*}$ Sung-Ho Ha, Hye Min Lee, and Jong-Chul Kim \\ Department of Chemistry and Carbohydrate Bioproduat Research Center, Sejong Lniversity, Seoul 143-747, Korea \\ ${ }^{+}$E-mail: sschoilà sejong.ac:kr \\ Recened Febriary 17,2007
}

Key Words : Silver cluster, Silver salt, Desorption, Ionization, LDI-TOFMS

Matrix-assisted laser desorptionitionization time-of-llight mass spectrometry (MALDI-TOFMS) has been used to characterize a wide range of polymers. ${ }^{1-5}$ In a MALDI mass spectrum, the protonated molecule and cation adduct of an analyte give an important information about the molecular ion mass. Some researchers reported that sliver clusters can be effectively produced under MAL.DI conditions from various silver salts in the presence of various matrices. ${ }^{(1-9)}$ During the analysis of organic molecules when $\Lambda \mathrm{g}^{\prime}$ is used as the cationization agent, it can be recognized that besides the desired adduct ions, $[\mathrm{M}+\mathrm{Ag}\rceil^{\prime}$, an additional silver cluster ions, $\mathrm{Ag}_{\mathrm{g}}$ is lomed. Kcki and coworkers ${ }^{7}$ studied the matrix effect on the formation of the silver cluster ions under $\mathrm{MAl}$.DI conditions using reductive polar organic matrices and silver trifluoroacetate (AgTFA) and reported that the matrix greatly influenced the resulting cluster ion abundances.

Metal clusters are very important in both theoretical and practical points of view. Investigation of cluster formation in mass spectrometry provides valuable information on the stability and electronic properties of clusters of different size. Studies on the formation of silver cluster ions under MAL.DI conditions have been performed in the presence of matrices such as 2,5-dihydroxybenzoic acid (DHB), 3,5dimethoxy-4-hydroxycinnamic acid (sinapinic acid, SA). trans-3-indoleacrylic acid (IAA), 2-(4-hydroxyphenylazo)benzoic acid (HABA), all-trans-retinoic acid (RTA). and 1,8,9-trihydroxyanthracene (dithranol, DIT). ${ }^{7-10}$ Formation of silver cluster ions directly from silver salts in the absence of matrix was not studied. In the present work, we investigated the silver cluster ions formed directly from silver salts of silver benzoate (AgBz), AgTFA, silver nitrate ( $\mathrm{AgNO}$ ), and silver $p$-toluenesulfonate (AgTS) in the absence of any matrices. Scheme 1 shows their chemical structures.

Figures 1, 2, 3, and 4 are the laser desorption/ionization (I.DI) mass spectra of $\mathrm{AgB}_{7}$, AgTFA, $\mathrm{AgNO}_{3}$, and AgTS. respectively. Only the mass spectrum of $\mathrm{AgB}$. shows a clear distribution of the silver cluster ions. It is a surprising result that the silver cluster ions are directly formed from $\mathrm{AgB}$. in the absence of any matrix. Macha and coworkers" investigated the formation of silver clusters from silver salts such as $\mathrm{AgTFA}, \mathrm{AgNO}_{3}$, and silver acetylacetonate [Ag(acac)]. but silver clusters were not detected. The cluster ion intensity distribution of $\mathrm{AgBz}$. shows an odd-even alternation pattem as shown in Figure 1 . In other words, the intensity of the odd-number cluster ions tends to be stronger than that of the neighboring even-number cluster ions, especially in the low-mass range. The mass spectrum also shows a steep decrease of the ion intensities after some cluster ions called magic numbers. Figure 1 shows clear magic numbers of $n=$ 9 and 21 of $\mathrm{Ag}_{n}$. The specilic ion intensity distribution of the silver cluster ions can be explained with the theoretical models. ${ }^{10-1,3}$ Since odd-number silver cluster ions have even numbers of valance electrons that become paired in the electronic shell of the formed clusters, they show stronger stabilities and higher ion intensities compared to those of even-number ones which have odd-number of electrons in their electronic shells. In addition, odd-number silver cluster ions tended to lose two silver atoms in their dissociation while even-number clusters lose a single atom. ${ }^{14}$ The magic numbers are in good agreement with the jellium model theory. $^{13.15}$ In the jellium model ${ }^{15.16}$ one assumes that the positive ions of the cluster are smeared into a uniform background of spherical shape with density equal to that in the bulk. The electrons respond to this uniform background and fill the successive angular momentum states in accordance with the rules of guantum mechanics. A cluster shows pronounced stability when one of the angular momentum levels is filled. This shell closure occurs for alkali atoms at $n$ $=2,8,20,40,58,92, \ldots$

Formation of the silver cluster ions has been studied in the presence of matrix. ${ }^{7-10}$ In general, formation of the silver

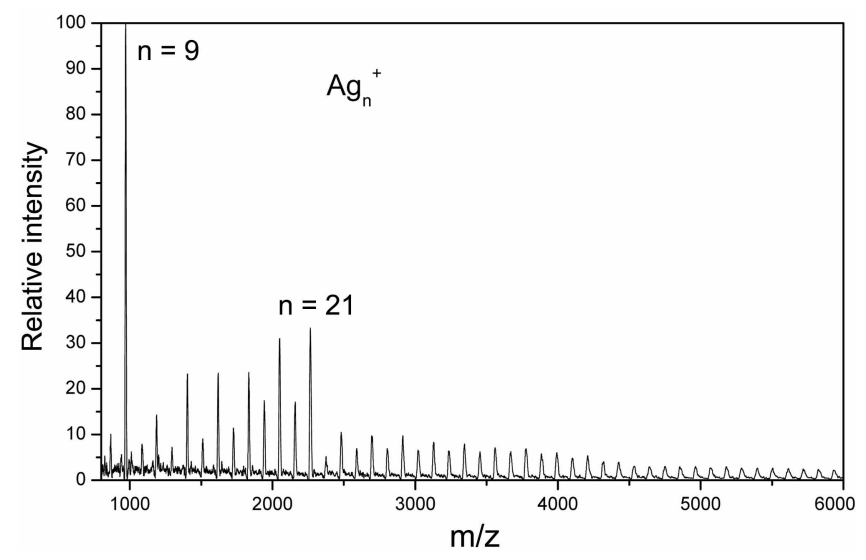

Figure 1. 1.DJ-TOF mass spectrum of $\mathrm{AgB}$. 


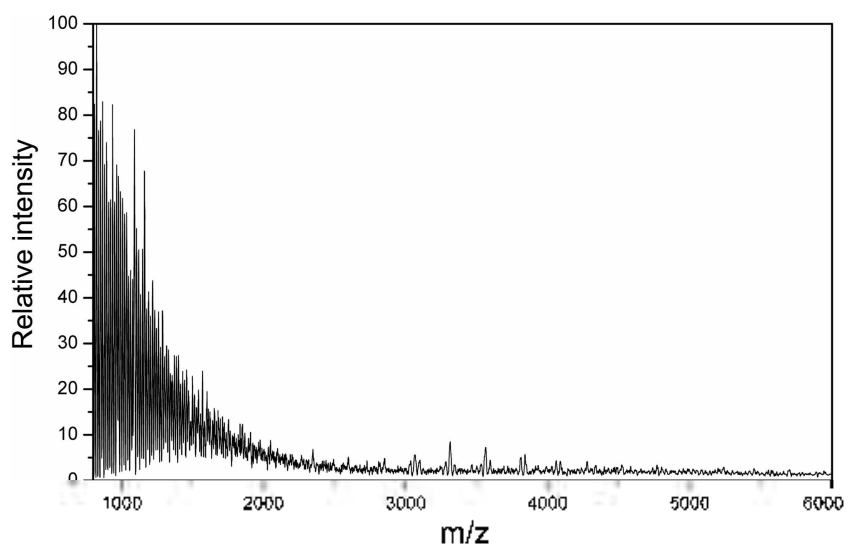

Figure 2. LDI-TOl mass spectrum of $\Lambda \mathrm{glTA}$.

cluster ions in the presence of matrix can be explained with a gas phase reaction. ${ }^{7.10}$ Laser desorption of silver-containing salts produces large amounts of the gas phase silver atoms. After their formation, the silver atoms undergo clustering reactions to form larger clusters. Kéki and coworkers ${ }^{7}$ suggested that neutral silver clusters could be formed through two different processes. The first is the charge transfer reaction of silver ions with the ground or excited neutral matrix molecules. The other is the free electron, which is generated from the matrix molecule by photoionization, capture reaction of silver ions.

AglifA has been commonly used as a silver salt for the studies on the formation of silver cluster ions in the presence of matrices and the specific ion intensity distribution of the silver cluster ions has been also reported..$^{7-10}$ However, the mass spectrum of Ag'HA in the absence of matrix does not show any silver cluster ions as shown in figure 2 . The other silver salts of $\mathrm{AgNO}$; and $\mathrm{AgTS}$ can not also generate silver cluster ions in the absence of matrix as shown in Figures 3 and 4 , respectively. Of the silver salts, only $\mathrm{AgBz}$ can generate the silver cluster ions in the absence of matrix by laser desorption/ionization.

In order to generate ions by laser desorption/ionization, an analyte should absorb the laser light. The silver salt should absorb the laser frequency to form silver cluster ions since in

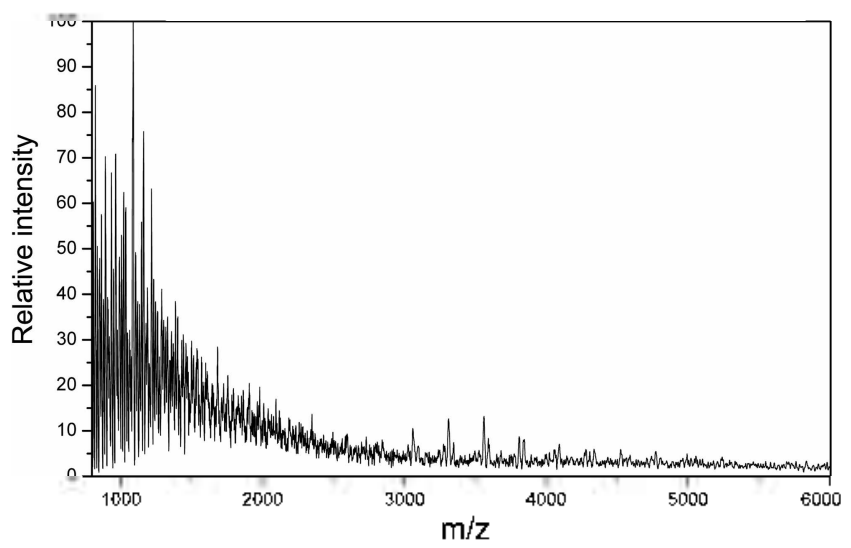

Figure 3. I,I)]-1 OF mass spectrum of $\mathrm{AgNO}_{3}$.

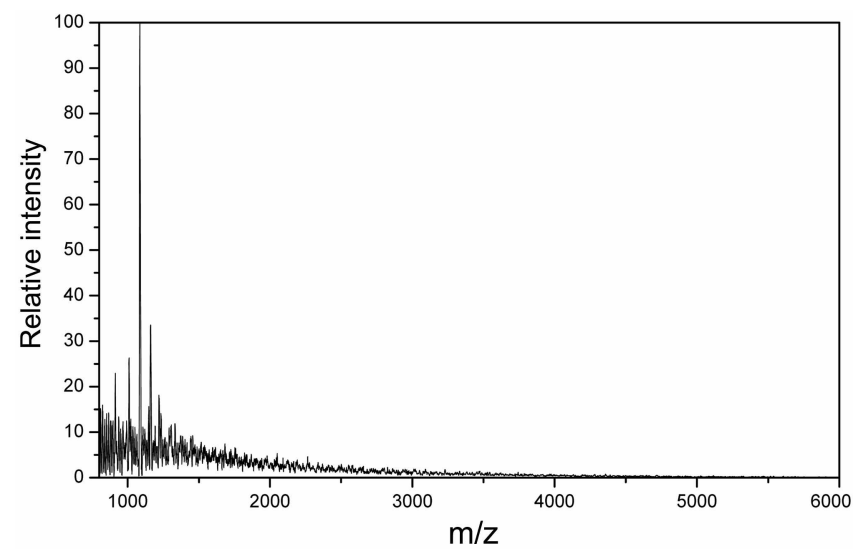

Figure 4. LDI-TOJ' mass spectrum of $\mathrm{Ag} \mathrm{IS}$.

this work we do not use any matrices which can absorb the laser frequency. UV absorption spectra of the silver salts (AgBz, Ag]FA, $\mathrm{AgNO}_{3}$, and AgTS) obtained in water solution were examined. All the silver salts used in the present study do not absorb $337 \mathrm{~nm}$ of nitrogen laser light. UV spectrum of benzoic acid was also investigated since benzoic acid is the conjugate acid of benzoate anion formed by dissociation and hydrolysis in water from silver benzoate. The benzoic acid also does not absorb $337 \mathrm{~nm} .{ }^{17.18}$ Powder silver benzoate was analyzed using LDI-JOHMS to investigate the formation of silver cluster ions in powder state since UV absorption of the silver benzoate solution will not be different with that of the solid silver benzoate. The powder sample for LD]-[OHMS analysis was prepared by putting the grinded silver benzoate powder on the double-stick taped on the sample plate. LDI-]OF mass spectrum of the powder silver benzoate was obtained, but the spectrum did not show any silver cluster ions. This implies that the powder silver

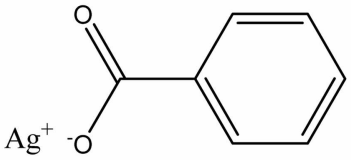

(a)

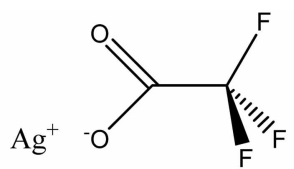

(b)<smiles>O=[N+]([O-])[O-]</smiles>

(c)

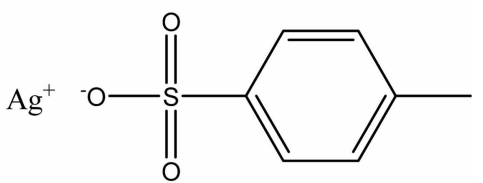

(d)
Scheme 1. Chemical structures of silver salts. (a) silver benzoale $(\mathrm{AgBz})$, (b) silver trifluoroacetate (AgTFA), (c) silver nitrute $(\mathrm{AgNO})_{i}$. and (d) silver $p$-toluenesulfonate (AgTS).

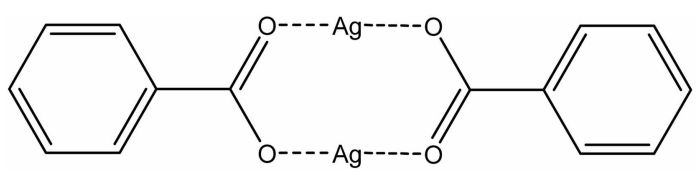

Scheme 2. Pussible structure of the two silver benzoate molecules. 
benzoate does not also absorb $337 \mathrm{~lm}$.

Absorption of the nitrogen laser light and formation of the silver cluster ions can not be clearly explained. Further study for that is needed. We only believe that the crystalline structure of silver benzoate can affect the formation of silver clusters. Crystallization of silver benzoate is processed by evaporation of water from the silver benzoate solution. It is possible that the silver benzoate has the crystalline structure of two molecules as shown in Scheme 2. Silver clusters can be pre-formed in the condensed phase due to photochemistry or other chemical processes. followed by laser desorption of the pre-formed clusters. If the crystalline silver benzoate absorbs the laser light. silver benzoate molecules will be desorbed and silver cluster and benzoyl peroxide can be formed by reaction of them. Synthesis of silver clusters using silver benzoate has been reported. ${ }^{19,31}$ Yin and coworkers $^{21}$ reported that silver adduct of two acetyl benzoyl peroxide molecules was decomposed to the silver adduct of one benzoyl peroxide. Silver clusters can absorb the nitrogen laser light.

\section{Experimental Section}

Silver benzoate $(\mathrm{AgBz})$. silver trifluoroacetate (AgTFA). silver nitrate $\left(\mathrm{AgNO}_{3}\right)$, and silver $p$-toluenesulfonate (AgTS) of Aldrich $\mathrm{Co}$. were employed as silver salts. The silver salts were dissolved in distilled water and the concentrations were $0.01 \mathrm{M}$. The solution of $1 \mu \mathrm{L}$ was spotted onto the sample plate and dried. Mass spectra were obtained with AximaLNR MALDI-TOFMS (Kratos-Shimadzu Co. of Japan). Ions were produced by irradiation of the sample with nitrogen laser $(337 \mathrm{~nm})$. Profiling of product ions was achieved in the positive mode using linear TOF. The accelerating voltage was $20 \mathrm{kV}$. The sum of 50 shots was collected for each spectrunt.

\section{References and Notes}

1. Cha. S: Kim. H.-T. Bull Kor Chem. Soc. 2003. 2f. 1308.

2. Park. S.-J.: Park, D.-H.: Sul, S.; Oh, S; Park. I.S.; Chung. D. S.; Kim. H.-J ; Kim. M.S.; Lee, S.-W. Bull. Kor: Chem. Soc. 2004. 25. 1791 .

3. Moon. J. H.: Yoon. S. H.: Kim. M. S. Bull. Kor Chem. Soc. 2005. 26. 763.

4. Choi. S.-S.: Ha. S.-H. Bull. Kor Chent Soc. 2006.27 .1243

5. Pittenauer, E.: Zell. M.; Belgacem, O.: Raptakis. E.: Mistrik. R: Allmaier. G. J. Mass Spectrom. 2006. $11,421$.

6. Rashidzadeh, H.; Guo. B. Chem. Plys. Lett. 1999, 310, 466

7. Kéki. S.: Szilágyi. S. L.: Török. J.: Deák. Gy.: Zsuga. M. J. Phns. Chent. B 2003. 107. 4818 .

8. Kéki. S.: Nagy. L.: Deák. Gy.: Zsuga. M.: Somogyi. L.: Lévai. A Am. Chem. Soc. Mass Spectron. 2004. 15.879.

9. Macha. S. F.: Limbach, P. A.; Hanton. S. D.: Owens, K. G. J. Am Soc. 1 Iass Spectrom $2001,12,732$.

10. Rashidzadeh. H.: Guo. B. Chem. Phys. Lett. 1999. 310.466

11. Balbas. L. C.: Rubio. A.: Alonso. J. A. Chen. Phws 1988. 120. 239

12. Katakuse, I; Ichilhara. T.: Fujita, Y: Matsuo, T.: Sakurai, T,; Matsuda, H. Int. I. Hass Spectrom. Ion Processes 1986. 74. 33

13. Knight, W. D.: Clemenger. K; de Heer, A. W.: Saunder, A. W: Chou. M. Y.: Cohen. M. L. Phis Rev: Lett. 1984. 52.2141.

14. Krucheberg. S.: Dietrich. G.: Lutzenkirchen. K.: Schweikhard. L.: Walther. C. Ziegler. J. Int. J. Mass Sipectron. Ion Processes 1996. 155. 141 .

15. Magaud. L.; Khanna. S. N.; Jena. P. Chem. Phys. Lett. 1991. 183. 333 .

16. De Heer. W. A.: Knight, W. D.; Chou, M. Y.: Cohen. M. L. Solid State Phis. 1987. 40.93 .

17. Saada. B.: Baria. F.: Saleha. M. I.: Alumadb. K.: Talibb. M. K. M. J. Chrontatogr \& 2005. 1073. 393

18. Lund Myhre. C. E.: Nielsen. C. J. Amos. Chem. Phos. 2004, 4. 1759 .

19. Chaki, N. K: Sudrik. S. G.; Sonawane. H. R:; Vjayamohanan. K. Chent Conmum 2002.76

20. Chitsaz. S.: Fenshe. D.: Fuhr. O. Angew. Chem. Int Ed 2006. 45. 8055 .

21. Yin. H.: Hachey, D. L.: Porter. N. A. J. Am. Soc. Mass Spectron 2001. 12,49 